\title{
Generalized square isometries - an improvement for fractal image coding
}

\author{
Dan C. Popescu ${ }^{1}$, Alex Dimca ${ }^{2}$ and Hong Yan $^{1}$ \\ 1 Department of Electrical Engineering, Sydney University, Sydney 2006, Australia \\ ${ }^{2}$ Department of Mathematics, Sydney University, Sydney 2006, Australia
}

\begin{abstract}
Most recent advances in fractal image coding have been concentrating on better adaptive coding algorithms, on extending the variety of the blocks and on search strategies to reduce the encoding time. Very little has been done to challenge the linear model of the fractal transformations used so far in practical applications. In this paper we explain why effective non-linear transformations are not easy to find and propose a model based on conformal mappings in the geometric domain that are a natural extension of the affine model. Our compression results show improvements over the linear model and support the hope that a deeper understanding of the notion of self-similarity would further advance fractal image coding.
\end{abstract}

\section{Introduction}

Barnsley's iterated function systems (IFS) and the collage theorem have provided the motivation for a class of fractal image compression techniques [1]. However, the collage theorem gives no hint of how to find an IFS that would approximate a given image and in general, a coverage of an image in terms of contracted copies of itself under reasonably simple transforms seems unlikely to exist.

The first practical approach to fractal image compression was proposed by Jacquin [2]. With this method, the image is split in small square blocks that tile the image, called range blocks. A pool of larger size square blocks, called domain blocks, that may be located anywhere in the image is also created a priori. The IFS consists of a set of transformations - one per range block. Every such transformation tries to match the range block with a domain block under a mapping that is a composition of a scaling from domain size to range size, a square isometry and a linear transformation in the gray level. This is a main departure from the spirit of the collage theorem: the image is covered with contracted copies of larger parts of the image, rather than the image itself. In practical terms, this search for a distributed self-similarity at microscopic level increases the chances for finding a good fractal code. It also has the negative effect of leading to long encoding times, to a point where, with any attempt to extend the model, a mechanism for efficient block matching becomes imperative.

Focusing our attention on one domain block - range block transformation, we notice that it is the composition of two linear transformations, one in the gray level domain and the other in the geometric domain. It is normal to ask 
whether the use of 3 bits only for the geometric transformation ( 8 isometries of the square) and some 12 to 16 bits for the gray level one represents an optimal code allocation.

In this paper we investigate the virtually unexplored field of non-linear transformations and address the question of whether increasing the number of geometrical transforms would increase the probability of a good match for larger blocks to a point where it would compensate for the extra code and result in higher overall compression ratios.

\section{Generalized square isometries}

Several attempts with continuous and one-to-one mappings which were not smooth have failed to improve the compression ratio, those transformations being "naturally rejected". We turned our attention to conformal mappings, which produce no angular distorsion.

If the range and domain blocks were disks, not squares, the geometric transformations could be extended by allowing rotations of arbitrary angles, rather than multiples of $90^{\circ}$. Nevertheless, a square can be mapped into a disk with minimal distortion and preserving angles. This would further allow the construction of transformations that rotate squares into squares with arbitrary angles. It is easier to understand the continuous model of this transformation acting in the complex plane $\mathbb{C}$ as the functions involved have a simpler form as functions of a complex variable. We denote by $\mathbb{Z}$ the set of all integers, by $i$ the imaginary unit $\left(i^{2}=-1\right)$ and also make the following notations for some subsets of $\mathbb{C}$ :

$\mathbb{C}_{-}=\{z \mid z \in \mathbb{C}, \operatorname{Im} z \leq 0\}$, the lower semiplane;

$\mathcal{D}=\{z|z \in \mathbb{C}| z \mid, \leq 1\}$, the unit disk and

$\mathcal{S Q}=\{z \mid z \in \mathbb{C}, 0 \leq \operatorname{Re} z \leq 1,0 \leq \operatorname{Im} z \leq 1\}$, the unit square.

The Weierstrass $\wp$ function:

$$
\begin{aligned}
\wp & : \mathcal{S Q} \mapsto \mathbb{C}_{-} \\
\wp(z) & =\sum_{k, l \in \mathbb{Z}} \frac{1}{(z-2 k-2 l \cdot i)^{2}}
\end{aligned}
$$

is a conformal one-to-one mapping of the unit square into the lower semiplane [5]. Denote by $z_{0}=\wp(0.5+0.5 i) \approx-1.718796 i$ and by $\overline{z_{0}}$ the complex conjugate of $z_{0}$. Then:

$$
\begin{gathered}
\omega: \mathbb{C}_{-} \mapsto \mathcal{D} \\
\omega(z)=e^{\frac{3 \pi}{4} i} \cdot \frac{z-z_{0}}{z-\overline{z_{0}}}
\end{gathered}
$$

is a one to one conformal mapping of $\mathbb{C}_{-}$into the unit disk. It follows that

$$
\begin{aligned}
& \varphi: \mathcal{S Q} \mapsto \mathcal{D} \\
& \varphi=\omega \circ \wp
\end{aligned}
$$


is a one-to-one conformal mapping of the unit square into the unit disk. The effects of $\varphi$ are illustrated in Figs. 1 and 2, where the square of Fig. 1 is mapped into the disk of Fig. 2 by $\varphi$.

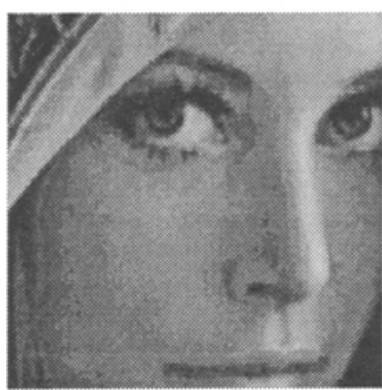

Fig. 1: Initial square

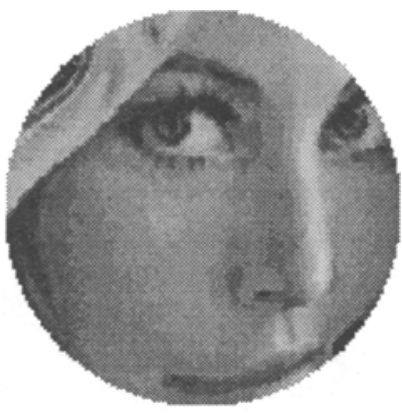

Fig. 2: Mapping of Fig. 1 by $\varphi$

For any angle $\theta, \theta \in\left[0,360^{\circ}\right)$ we denote by $r_{\theta}$ the usual clockwise rotation of angle $\theta$ :

$$
\begin{aligned}
r_{\theta} & : \mathcal{D} \mapsto \mathcal{D} \\
r_{\theta}(z) & =e^{-\frac{\pi \theta}{180} i} \cdot z
\end{aligned}
$$

A square rotation of angle $\theta$ is defined as the transformation

$$
\begin{aligned}
& s q r_{\theta}: \mathcal{S Q} \mapsto \mathcal{S Q} \\
& s q r_{\theta}=\varphi^{-1} \circ r_{\theta} \circ \varphi
\end{aligned}
$$

Figs. $5-7$ show the results of mapping the square of fig. 1 by square rotations of $30^{\circ}, 55^{\circ}$ and $70^{\circ}$.

Square antirotations are defined similarly by compositions with a flip over the real axis. Finally, we define the set $\mathcal{I}$ of generalized square isometries as the set of all square rotations and antirotations.

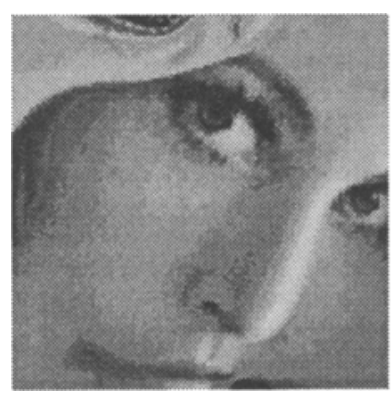

Fig. 3: Mapping of Fig. 1 by $r s q_{30^{\circ}}$

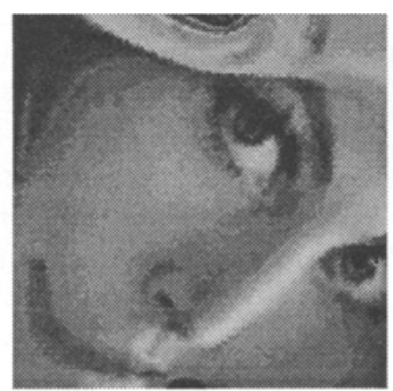

Fig. 4: Mapping of Fig. 1 by $r s q_{55^{\circ}}$

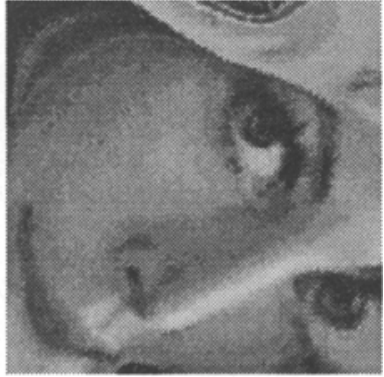

Fig. 5: Mapping of Fig. 1 by $r s q_{70^{\circ}}$ 


\section{The discrete model}

\subsection{Discrete generalized square isometries}

Only a finite number of generalized square isometries will be used in practice. They are calculated only once according to the previous formulas and the hardcoded patterns of their discrete equivalents are known both at the encoder and the decoder. To understand how these discrete hardcoded patterns are generated without going into unimportant details we shall consider an analogy with classical isometries. A $90^{\circ}$ rotation of a discrete block of $n \times n$ pixels is given by the mapping

$$
(x, y) \mapsto(n-1-y, x)
$$

It says that the new pixel at $(0,0)$ is computed from the old pixel at $(0, n-1)$, etc. If the mapping is followed by a spatial contraction of (say) factor 2 , from a $2 n \times 2 n$ domain block to a $n \times n$ range block we would have that the gray level of the pixel at $(0,0)$ is obtained by averaging the pixels at $(0,2 n-1),(0,2 n-2),(1,2 n-1),(1,2 n-2)$, each with the weight 0.25 . It is clear that the transformation can be described either by giving the set of "weighted lists" or by applying formula (6) and both approaches are computationally fast. The "weighted list" approach works with the formulas of chapter 2 as well even if the "real-time formula" approach similar to (6) would be impractical in terms of computational complexity.

\subsection{A filtering mechanism}

The critical part of this fractal image compression technique is the encoding process. For each range block, it requires a search through the pool of domain blocks for the best match under all available transformations. If we denote by $N_{r}, N_{d}, N_{t}$ the number of range blocks, the number of domain blocks and the number of transformations, then an exhaustive search has a computational complexity $O\left(N_{r} \cdot N_{d} \cdot N_{t}\right)$.

However, with the method we propose, moment features can be defined for each block. They are computationally unexpensive and they have to be calculated only once for each block - which means a complexity $O\left(N_{r}+N_{d}\right)$. The first order moments define the barycenter orientation which is invariant to a linear gray scale mapping and moves consistently with the geometric transformations. Only the best geometric transformations are attempted, typically 2 per range block/domain block pair. If the domain block and the geometric transformation are fixed, the parameters of the linear transformation in the gray level can easily be found by an autoregression formula [3]. Because of this tight control, the increased number of transformations does not result in a significantly increased computational complexity. The search complexity is reduced to $O\left(N_{r} \cdot N_{d}\right)$.

We use a generalization of an idea presented in [5] to further reduce the search for a matching domain block. We define two moment features, $\mathcal{M}_{1}$ and $\mathcal{M}_{2}$, associated with (the disk equivalent $\mathcal{D}$ of) each range and domain block as:

$$
\mathcal{M}_{1}(\bar{b})=\frac{\iint_{\mathcal{D}} \frac{g(x, y)-m(\bar{b})}{\operatorname{dev}(\bar{b})} \cdot\left(x^{2}+y^{2}\right) d x d y}{\iint_{\mathcal{D}} d x d y}
$$




$$
\mathcal{M}_{2}(\bar{b})=\frac{\iint_{\mathcal{D}} \frac{g(x, y)-m(\bar{b})}{\operatorname{dev}(\bar{b})} \cdot\left(x^{2}+y^{2}\right)^{-\frac{1}{4}} d x d y}{\iint_{\mathcal{D}} d x d y}
$$

where $m(\bar{b}), \operatorname{dev}(\bar{b})$ are the mean and standard deviation of block $\bar{b}$ and $g(x, y)$ is the gray scale level at position $(x, y)$. The absolute values of $\mathcal{M}_{1}$ and $\mathcal{M}_{2}$ are invariant under any linear transformation in the gray level and any geometric mapping of $\mathcal{I}$. When looking for a good match for a given range block, only domain blocks with similar features would be investigated. Discrete equivalents of $\mathcal{M}_{1}$ and $\mathcal{M}_{2}$ that are computationally unexpensive can be defined.

\section{Experimental results}

We use an adaptive coding scheme that splits the image into non-overlapping square range blocks, according to a tree structure. This tree structure is more flexible than a quadtree, with smoother transitions in block sizes [5]. It uses $4 \times 4$, $6 \times 6,8 \times 8$ and $12 \times 12$ size range blocks.

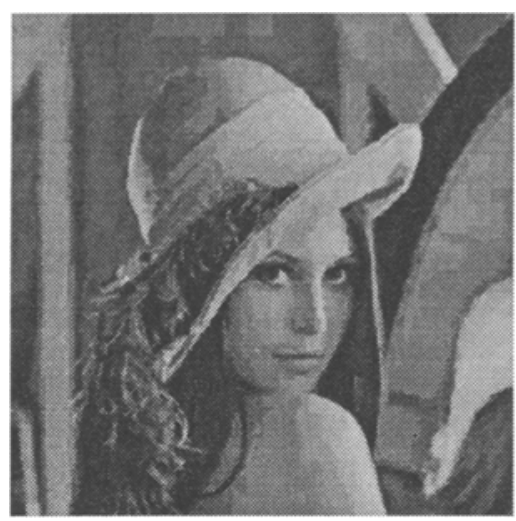

Fig. 6: Decoded image using 8 classical isometries

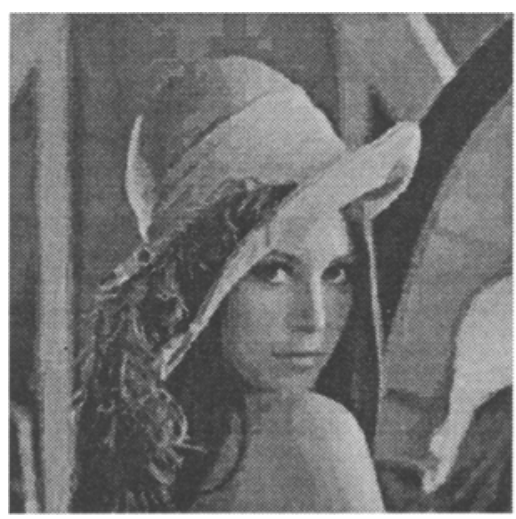

Fig. 8: Decoded image using gen. square isometries

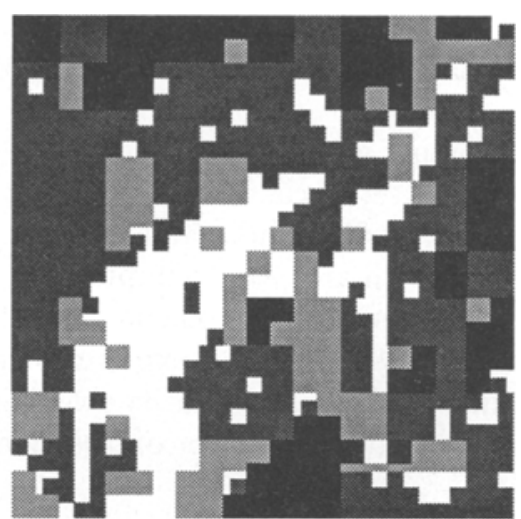

Fig. 7: Range block distribution for Fig. 6

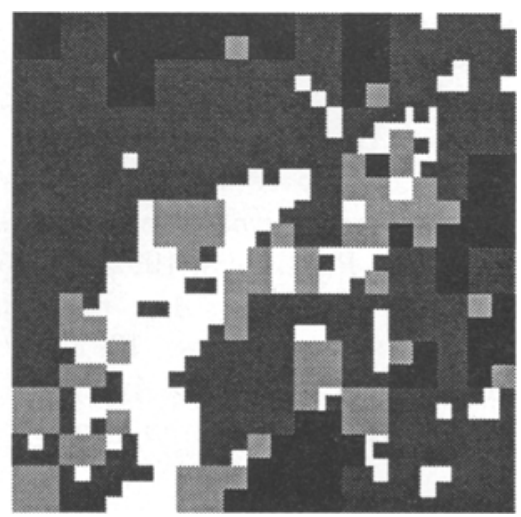

Fig. 9: Range block distribution for Fig. 8 
Figs. 6 and 8 show two compression results of the $256 \times 256$ Lena image using this adaptive partitioning scheme. For the reconstruction of Fig. 6 only the 8 classical affine transforms have been used for all range blocks. The reconstruction of Fig. 8 was obtained using $8,16,32$ and 32 generalized square isometries for $4 \times 4,6 \times 6,8 \times 8$ and $12 \times 12$ size range blocks, respectively. The PSNR for both reconstructions is $32.8 \mathrm{~dB}$. Since the quality of the reconstruction is pretty high, the PSNR is a good measure of the visual degradation, so we can consider the two reconstructions of equivalent quality. Figs. 7 and 9 display the range block allocation for the reconstructions of Figs. 6 and 8 , with $4 \times 4$ blocks colored in white, $6 \times 6$ blocks in light gray, $8 \times 8$ blocks in dark gray and $12 \times 12$ blocks in black. Intuitively, the darker the area, the higher is the (local) compression factor. It is noticeable that in Fig. 9 the darker areas "take over" the corresponding lighter ones in Fig. 7. Even if the extra bits necessary to encode more transformation reduce this gain, the compression factor for the reconstruction in Fig. 8 is 9.77 versus 8.97 for the reconstruction in Fig. 6 , an $8.9 \%$ improvement of the compression factor.

\section{Conclusions}

In this paper we have developed a non-linear model for fractal image compression based on a set of conformal mappings of the square into itself. A discrete model of these transformations and a filtering mechanism to reduce the encoding time, both of which are easy to implement, have been proposed. Our compression results with an adaptive scheme show improvements over the linear model. In a broader sense, the whole experiment gives ground to hope that a better understanding of the self similarity governing natural patterns could lead to further advances in fractal image compression.

\section{References}

1. Barnsley M.F.: Fractals Everywhere, Academic Press,Inc., 1988

2. Jacquin A.E.: Image Coding Based on a Fractal Theory of Iterated Contractive Image Transformations, IEEE Trans. on Image Processing,Vol. 1,No. 1, January 1992, pp. 18-30

3. Fisher Y., Jacobs E.W. and Boss R.D.: Fractal Image Compression Using Iterated Transforms, Image and Text Compression, J.A. Storer(ed.), Kluwer Academic Publishers, 1992, pp. 35-61

4. Frances K.: Complex Algebraic Curves, London Mathematical Society, Cambridge University Press, 1992

5. Popescu D.C., Yan H.: A Fractal Based Method for Color Image Compression, Journal of Electronic Imaging, vol. 4, nr. 1, January 1995, pp. 23-30 